\title{
GAIA Level 4 First Trimester Spontaneous Abortion
}

National Cancer Institute

\section{Source}

National Cancer Institute. GAIA Level 4 First Trimester Spontaneous Abortion. NCI

Thesaurus. Code C128779.

GAIA Level 4 First Trimester Spontaneous Abortion is defined by two criteria: first, it does not qualify as a Level 1, a Level 2, or a Level 3 First Trimester Spontaneous Abortion; second, a maternal self-report or documentation in the medical record of a pregnancy loss without sufficient ultrasound or laboratory evidence to confirm. 